\title{
TITLE:
}

\section{Limited slowdown of endocrine- disruptor diffusion in confined fluid lipid membranes}

$\operatorname{AUTHOR}(\mathrm{S})$ :

Okamura, E; Wakai, C; Matubayasi, N; Sugiura, Y; Nakahara, M

\section{CITATION:}

Okamura, E ... [et al]. Limited slowdown of endocrine-disruptor diffusion in confined fluid lipid membranes. PHYSICAL REVIEW LETTERS 2004, 93(24): 248101.

\section{ISSUE DATE:}

2004-12-10

URL:

http://hdl.handle.net/2433/50331

RIGHT:

Copyright 2004 American Physical Society 


\title{
Limited Slowdown of Endocrine-Disruptor Diffusion in Confined Fluid Lipid Membranes
}

\author{
Emiko Okamura, ${ }^{*}$ Chihiro Wakai, Nobuyuki Matubayasi, Yukio Sugiura, and Masaru Nakahara ${ }^{\dagger}$ \\ Institute for Chemical Research, Kyoto University, Uji, Kyoto 611-0011, Japan
}

(Received 14 May 2004; published 6 December 2004)

\begin{abstract}
Self-diffusion rates of lipids and trapped bisphenol A (BPA) are determined in various sizes of confined but fluid membranes by high-field-gradient NMR at $600 \mathrm{MHz}$. Micelles and vesicles of 3- to 400- $\mathrm{nm}$ diameters are used as model membranes to get an insight into the molecular diffusion in such soft environments. The slowdown of BPA and lipid motions is leveled off in 100- and 400-nm vesicles, although the hydrodynamic continuum model gives the aggregate motion slowed inversely to the aggregate size. Instead, the limited motion is related to the intra-membrane fluidity.
\end{abstract}

Molecular dynamics in confined geometries is an issue of physical, chemical, and biological significance [1]. It is still controversial how molecular diffusion is modified in such a confined but fluid environment. Zeolites and porous silica glasses are the model confined geometries most frequently used [1-4]. In these cases, confined spaces have been generally treated as solid, hard bodies with rigid diffusion boundaries. A biologically important confined system is the lipid bilayer membrane, a soft interface which separates the inside from the outside of a living cell. It is not a rigid but fluid space. Diffusion boundaries in a membrane are always fluctuating; they are not smooth but rough on the molecular level $[5,6]$.

Pulsed field gradient (PFG) NMR spectroscopy is a powerful technique to directly measure the molecular diffusion both for the host and guest species in a confined space. Its application to the membrane system has, however, been difficult because of the low signal resolution and slow diffusion. The recent PFG-NMR studies have used labeling or the magic-angle spinning technique to obtain well resolved NMR signals in membrane [7,8]. Here we first report the simultaneous diffusion measurement of lipids and trapped drugs in natural, freestanding membrane system, using high-field-gradient highresolution solution NMR at $600 \mathrm{MHz}$. Neither labeling nor spinning is used to avoid plausible artifacts. To get an insight into the molecular diffusion in a confined but soft space, we directly monitor and compare the selfdiffusions of an endocrine disruptor, bisphenol A (BPA, 4,4-isopropylidenediphenol), and lipid molecules in sizecontrolled micelles and vesicles as model membranes. To control the extent of boundary restriction in a confined space, we employ the micelles and vesicles with the average diameters of $3 \mathrm{~nm}$ (micelle), $30 \mathrm{~nm}$ [small unilamellar vesicle (SUV)], and 100 and $400 \mathrm{~nm}$ [large unilamellar vesicle (LUV)]. Here it should be noted that LUV is characterized by almost negligible surface curvature effect [9], in contrast to the highly curved micellar surface.

For the reliable monitoring of molecular motions in a highly viscous membrane environment [10], we have developed a specially designed PFG probe for a $600 \mathrm{MHz}$ apparatus in cooperation with JEOL (JEOL NM-60HXT5GR equipped with ECA600). In this probe, the field gradient (FG) coil is set closer than the other coils and the efficiency for generating the field is made maximal with a $30 \mathrm{~A}$ current. The field leakage is then minimized by placing a set of shield coils. The shield coils are designed through a numerical electromagnetic field calculation, and serves not only for the efficiency of field generation but also for preventing the deterioration of an observed spectrum. The probe can exert a field gradient up to $1350 \mathrm{G} / \mathrm{cm}$, while the maximum precision is attained at the medium power. The typical stack plot of the proton spectra is shown in Fig. 1.

The simultaneous monitoring of the drug and lipid movements allows us to explore how the drug mobility is related to the membrane lipid dynamics. Attention is also paid to the relation of the BPA mobility to the location in membrane previously determined by the static NMR analysis [11,12]. To ensure the site dependence of drug dynamics in membranes, we identify the location and the mobility of benzene and toluene in membrane and compare them with the case of BPA (hereafter, benzene and toluene are also called a "drug"). We also measured the aqueous solutions of BPA, benzene, and toluene for reference.

To ensure the reliability of our method, we first examine whether the high-field-gradient NMR measurement [13] reproduces the self-diffusion of membrane lipids in micelle, SUV, and 100- and 400-nm LUV. The lipid diffusion in membrane is, as listed in Table I, enhanced in the order of micelle $>$ SUV $>100-n m$ LUV $\approx$ 400-nm LUV, reflecting the surface curvature dependence of the dynamical structure [17]. Note that the surface curvature effect vanishes in 100- and 400-nm LUV. The lipid diffusion rate in LUV corresponds to the fluorescence recovery after photobleaching (FRAP) data [15] and the long-time molecular dynamics (MD) simulation [16]. The diffusion rate in SUV is almost analogous to the PFG-NMR result of dioleoylphosphatidylcholine (DOPC) SUV [14]. The lipid diffusion in 


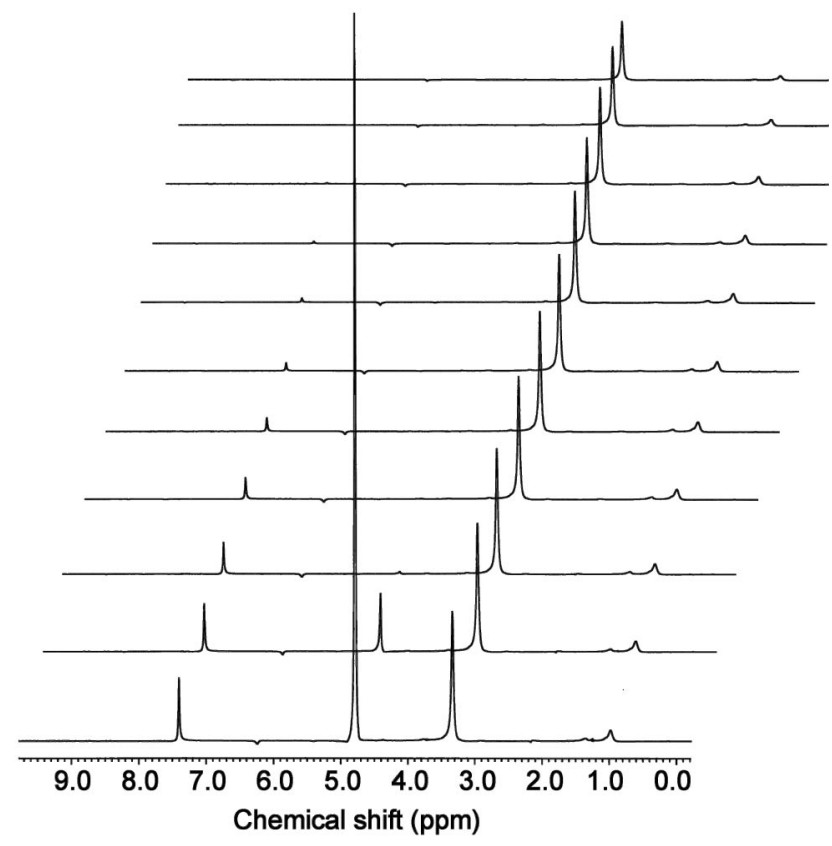

FIG. 1. Typical stack plot of the ${ }^{1} \mathrm{H}$ NMR spectra in the PFG measurement. Here benzene is incorporated into 100-nm LUV to show no signal overlap of the drug and lipid in the spectrum. The signals are assigned to benzene (7.4 ppm), water (4.8 ppm), lipid choline methyl (3.3 ppm), and lipid chain methyl (1.0 ppm).

micelles is typical of that in surfactant micelles [18]. All results confirm the reliability of our method.

To further confirm the validity of our approach, we examine whether the appropriate self-diffusion coefficients can be obtained for the aqueous BPA solution [19]. The self-diffusion coefficient of BPA in water is $6.0 \times 10^{-10} \mathrm{~m}^{2} \mathrm{~s}^{-1}$ at $30^{\circ} \mathrm{C}$, almost half of the diffusion rate of benzene in water, $1.1 \times 10^{-9} \mathrm{~m}^{2} \mathrm{~s}^{-1}$ at $30^{\circ} \mathrm{C}$ [20]. The result is reasonable in view of the BPA structure with two benzene rings.

Next we directly monitor the diffusion rates [21] of the trapped BPA in micelles, SUV, and LUV. As listed in Table II, the self-diffusion coefficients of BPA in the confined media are ten to 50 times slower than that in water. The BPA diffusion is dominated by the membrane particle size. It is noted that the BPA motion is almost unaltered in 100- and 400-nm LUV in comparison to that in SUV; recall that the surface curvature effect in LUV can be neglected on the molecular level.

To see whether the BPA mobility is related to membrane lipid dynamics, the self-diffusion coefficient of the lipid molecules is simultaneously determined. As summarized in Table II, the BPA mobility is comparable to the respective lipid diffusion; it is synchronized or concerted with the lipid matrix motion. The concerted movement means that the molecular diffusion in membrane is dominated by the dynamic property of the membrane matrices.

In contrast, benzene and toluene are 10 and 6 times as mobile as the membrane lipids, respectively, as summarized in Fig. 2. The movements of benzene and toluene are more independent of the membrane lipid motions, in marked contrast to the concerted mobility of BPA in membrane. The motional slowdown of BPA in membrane is notable in view of the BPA structure corresponding to two molecules of toluene.

To see why the BPA motion is concerted with membrane lipids, we compare the locations of BPA, toluene, and benzene in LUV by an atomic-level ${ }^{13} \mathrm{C}$ NMR analysis $[23,24]$. As illustrated by Fig. 2, BPA has a high affinity for the membrane interface [12]. The BPA motion is slowed down by the site-specific, strong binding to

TABLE I. Self-diffusion coefficient of lipids in lysophosphatidylcholine micelle, egg phosphatidylcholine SUV, and LUV determined by the PFG-NMR method. The lipid concentrations are fixed to $30 \mathrm{mM}$. Diffusion rates of lipid headgroup and hydrophobic core regions are independently determined from the choline methyl signal and the chain methylene or methyl signal, respectively. Diffusion coefficients of lipids, estimated from the FRAP method and MD simulation, are also listed.

\begin{tabular}{|c|c|c|c|c|c|}
\hline & \multirow[t]{2}{*}{ Method } & \multicolumn{3}{|c|}{ Self-diffusion coefficient $\left(10^{-10} \mathrm{~m}^{2} \mathrm{~s}^{-1}\right)$} & \multirow[t]{2}{*}{ Reference } \\
\hline & & Headgroup & & Chain core & \\
\hline In micelle $(3 \mathrm{~nm})^{\mathrm{a}}$ & PFG-NMR & $0.65 \pm 0.02$ & $0.5-1.3^{\mathrm{b}}$ & $0.63 \pm 0.02$ & $\begin{array}{l}\text { this Letter } \\
{[18]}\end{array}$ \\
\hline In SUV (30 nm) & $\begin{array}{l}\text { PFG-NMR } \\
\text { PFG-NMR }\end{array}$ & $0.24 \pm 0.01$ & $0.23-0.24^{\mathrm{c}}$ & $0.28 \pm 0.01$ & $\begin{array}{l}\text { this Letter } \\
{[14]}\end{array}$ \\
\hline In LUV (100 nm) & PFG-NMR & $0.12 \pm 0.01$ & & $0.14 \pm 0.01$ & this Letter \\
\hline In LUV (400 nm) & $\begin{array}{l}\text { PFG-NMR } \\
\text { FRAP } \\
\text { MD }\end{array}$ & $0.13 \pm 0.01$ & $\begin{array}{l}0.124-0.134^{\mathrm{d}} \\
0.12^{\mathrm{e}}\end{array}$ & $0.15 \pm 0.01$ & $\begin{array}{l}\text { this Letter } \\
{[15]} \\
{[16]}\end{array}$ \\
\hline
\end{tabular}

${ }^{\mathrm{a}}$ In parentheses are the average diameters of the particles determined from the quasielastic light scattering.

${ }^{\mathrm{b}}$ Summarized values for ionic surfactants at $30^{\circ} \mathrm{C}$.

${ }^{\mathrm{c}}$ For DOPC SUV at $25^{\circ} \mathrm{C}$.

${ }^{\mathrm{d}}$ For DOPC planar bilayer at $23^{\circ} \mathrm{C}$.

${ }^{\mathrm{e}}$ For DPPC bilayer at $60^{\circ} \mathrm{C}$. 
TABLE II. Self-diffusion coefficients of BPA in water and in model confined fluid membranes. The diffusion rates of the membrane lipid molecules are also shown. For the experimental conditions, see Table I. The HD diffusion rates [22] of the whole particle itself are listed in the right column.

\begin{tabular}{lllll}
\hline \hline & BPA & \multicolumn{2}{c}{$\begin{array}{c}\text { Self-diffusion coefficient }\left(10^{-10} \mathrm{~m}^{2} \mathrm{~s}^{-1}\right) \\
\text { Lipid }\end{array}$} & HD rigid sphere \\
& & Headgroup & Chain core & \\
In bulk water & $6.01 \pm 0.03$ & $\ldots$ & $\ldots$ & $0.63 \pm 0.02$ \\
In micelle & $0.55 \pm 0.01$ & $0.65 \pm 0.02$ & $0.27 \pm 0.01$ & 0.17 \\
In SUV & $0.26 \pm 0.01$ & $0.25 \pm 0.01$ & $0.14 \pm 0.01$ & 0.056 \\
In 100-nm LUV & $0.12 \pm 0.01$ & $0.12 \pm 0.01$ & $0.15 \pm 0.01$ & 0.014 \\
In 400-nm LUV & $0.12 \pm 0.01$ & $0.13 \pm 0.01$ & & \\
\hline \hline
\end{tabular}

a The HD estimate of the overall translational diffusion is $k_{B} T / 6 \pi \eta d$ with the HD radius $d$. The HD estimate to the overall rotational diffusion is $k_{B} T / 8 \pi \eta d^{3}$. This corresponds to an additional translational diffusion of $\sim k_{B} T / 8 \pi \eta d$ and does not change the magnitude order of the HD estimate listed.

membrane lipids. Benzene, in contrast, weakly interacts with the lipids; it is distributed from membrane interface to the hydrophobic chain region. The wide distribution of benzene in membrane is comparable to unrestricted and independent movements in lipid matrices.

It is significant to know how the motion of the whole lipid aggregate contributes to the molecular diffusion behavior. In Table II the diffusion rates of the lipid aggregates estimated from the hydrodynamic (HD) diffusion model [22] are summarized. The HD continuum

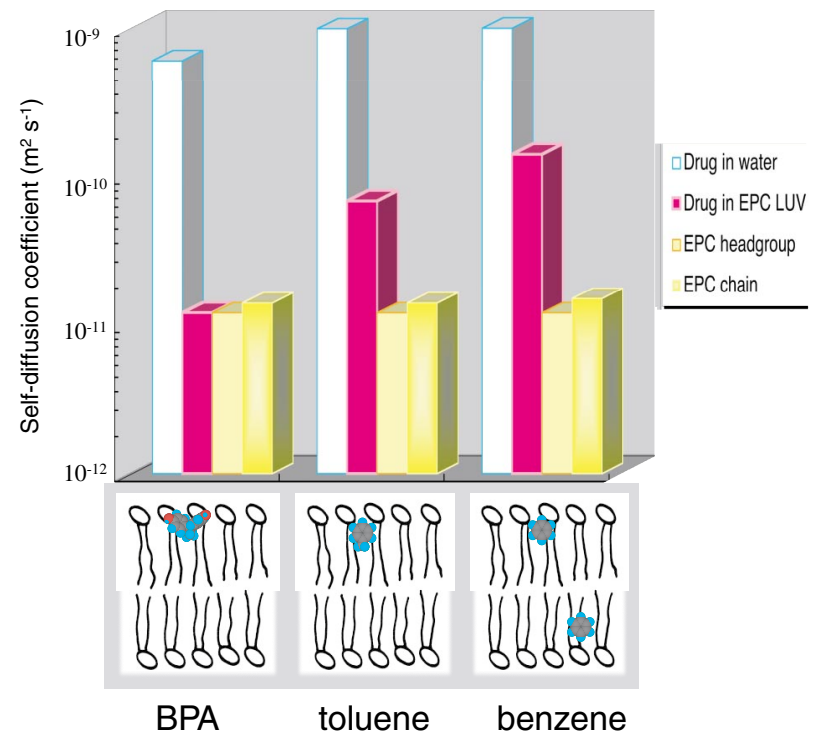

FIG. 2 (color). Mobility and location of BPA, toluene, and benzene in membrane. In the upper graph are the self-diffusion rates of these drugs in water (the white) and in 100-nm LUV (the red columns) at $30^{\circ} \mathrm{C}$. Mobility of membrane matrices is also expressed as the self-diffusion rates of both the lipid head group (the light yellow) and the chain core (the yellow columns). The location of the drug in the bilayer is shown schematically in the lower part. The drug is represented by the space filling model with $\mathrm{H}$, blue; $\mathrm{C}$, gray; and $\mathrm{O}$, red. picture applies to neither lipid nor BPA movement in the membrane. The point is that the slowdown of BPA and lipid motions is leveled off in 100- and 400-nm LUVs, although the HD continuum model gives the aggregate diffusion slowed down in inverse proportion to the aggregate size. The limited slowdown of BPA and lipid motions is due to the intra-membrane fluidity; no restriction is observed in sufficiently large membrane. The selfdiffusion of the lipid aggregate itself can be almost negligible on the molecular level and the intra-membrane motion dominates the observed diffusion coefficient. These are different from the diffusion of guest molecules in rigid zeolites which is influenced by crystallite boundary effects and intercrystallite contributions [4]. The motion of the whole micelle needs to be treated with care, in contrast, to analyze the diffusion coefficient for the micellar system. Table II shows that the intramembrane motion plays a significant role when the confined system is fluid and soft.

In summary, the site-selective, noninvasive solution NMR technique enables us to explore the dynamic property of the respective membrane component. The method opens a possibility to give insight into the wide range of molecular dynamics within a confined fluid membrane; neither labeling [25] nor the fluorescent probing [15] is required.

This work was supported by the Grant-in-Aid for Scientific Research 14540531 from Japan Society for the Promotion of Science and by the Grant-in-Aid for Creative Scientific Research 13NP0201, the Grant-in-Aid for Scientific Research on Priority Area 15076205, and the Grant-in-Aid for Exploratory Research 16655071 from the Ministry of Education, Culture, Sports, Science and Technology, Japan.

\footnotetext{
*Electronic address: emiko@scl.kyoto-u.ac.jp

${ }^{\dagger}$ Electronic address: nakahara@scl.kyoto-u.ac.jp
} 
[1] J. M. Drake and J. Klafter, Phys. Today 43, 46 (1990).

[2] J.-M. Zanotti, M.-C. Bellissent-Funel, and S. H. Chen, Phys. Rev. E 59, 3084 (1999).

[3] G. Rittig, C. G. Coe, and J. M. Zielinski, J. Am. Chem. Soc. 124, 5264 (2002).

[4] B. Geil et al., J. Chem. Phys. 116, 2184 (2002).

[5] M. C. Wiener and S. H. White, Biophys. J. 61, 437 (1992).

[6] H. I. Petrache et al., Phys. Rev. E 57, 7014 (1998).

[7] A. Filippov, G. Orädd, and G. Lindblom, Biophys. J. 84, 3079 (2003).

[8] H. C. Gaede and K. Gawrisch, Biophys. J. 85, 1734 (2003).

[9] R. Koynova and M. Caffrey, Biochim. Biophys. Acta 1376, 91 (1998).

[10] R. M. Venable et al., Science 262, 223 (1993).

[11] E. Okamura and M. Nakahara, J. Phys. Chem. B 103, 3505 (1999).

[12] E. Okamura, R. Kakitsubo, and M. Nakahara, Langmuir 15, 8332 (1999).

[13] The measurement was carried out at $30 \pm 0.1^{\circ} \mathrm{C}$ where lipid membrane is in the liquid-crystalline (fluid) phase. To avoid secondary effect by the intense signal of solvent water, the dante presaturation pulse sequence was used for the water resonance elimination. Rectangular FG pulses were applied with an interval $(\Delta)$ of $100 \mathrm{~ms}$ (solution) or $20 \mathrm{~ms}$ (membrane). To attenuate the spinecho signal, the pulse width $(\delta)$ was varied. The field gradient strength $G$ was fixed at $27.2 \mathrm{G} \mathrm{cm}^{-1}$ for solution measurements. For membrane, $G$ was increased to $222.0 \mathrm{G} \mathrm{cm}^{-1}$ which was sufficient for restricted diffusion rates. The intensity of a spin-echo signal is given by $A(\delta)=A(0) \exp \left\{-D \gamma^{2} G^{2} \delta^{2}(\Delta-\delta / 3)\right\}$, where $A(\delta)$ and $A(0)$ are the echo signal intensities when the field gradient is present and absent, respectively; $D$, the selfdiffusion coefficient; and $\gamma$, the gyromagnetic ratio of the proton $\left(2.675 \times 10^{8} \mathrm{rad} \mathrm{T}^{-1} \mathrm{~s}^{-1}\right)$. The diffusion coefficients were obtained by the least-square analysis of 6-10 experimental points of the echo signal attenuation. The experimental uncertainty was within $\pm 10 \%$. The drugs used in this study are preferentially partitioned into membrane because they are highly lipophilic. This is confirmed by no variation of the drug diffusion coefficient when the drug concentration is changed in the range from one to $25 \mathrm{~mol} \%$. Thus the drug diffusion between aggregates can be negligible on the experimental time scale. The trapped amount of the drug in membrane is under equilibrium, judging from the NMR signal before and after the diffusion measurement.

[14] P. Stilbs, G. Arvidson, and G. Lindblom, Chem. Phys. Lipids 35, 309 (1984).

[15] S. Ladha et al., Biophys. J. 71, 1364 (1996).

[16] E. Lindahl and O. Edholm, J. Chem. Phys. 115, 4938 (2001).

[17] E. Okamura et al., Chem. Lett. 1997, 1061 (1997).

[18] D. P. Bossev, M. Matsumoto, and M. Nakahara, Mixed Surfactant Systems, edited by M. Abe, (Marcel Dekker, New York, to be published).

[19] The solubility of BPA in water, determined from the ${ }^{1} \mathrm{H}$ NMR signal intensity, is $0.3 \mathrm{mM}$ at $30^{\circ} \mathrm{C}$. Our determination of $D$ illustrates the high sensitivity of the apparatus.

[20] M. Nakahara et al., J. Phys. Chem. 100, 1345 (1996).

[21] When the drug is trapped in the membrane for a sufficiently long time, the mean-square displacements of the drug and membrane molecules need to be coincident with each other in the long-time limit. The diffusion coefficients obtained in the present study are the ones through the fit to the equation appearing in Ref. [13]. In other words, our $D$ is an "effective" one conditioned by the experimental time scale. If the time scale is not long enough, the $D$ of the drug is not necessarily equal to the $D$ of the membrane molecule, while it is a certain measure of the molecular mobility in the aggregate.

[22] C. Wakai and M. Nakahara, J. Chem. Phys. 106, 7512 (1997).

[23] E. Okamura et al., J. Phys. Chem. B 105, 12616 (2001).

[24] E. Okamura and M. Nakahara, in Liquid Interfaces in Chemical, Biological, and Pharmaceutical Applications, edited by A. G. Volkov (Marcel Dekker, New York, 2001), p. 775 .

[25] J. H. Freed, Annu. Rev. Biophys. Biomol. Struct. 23, 1 (1994). 